\title{
Erratum to: En bloc transurethral resection with 2-micron continuous-wave laser for primary non-muscle-invasive bladder cancer: a randomized controlled trial
}

\author{
Xu Chen · Jun Liao $\cdot$ Lingwu Chen $\cdot$ Shaopeng Qiu $\cdot$ \\ Chengqiang Mo $\cdot$ Xiaopeng Mao $\cdot$ Yuanzhong Yang $\cdot$ \\ Shiying Zhou $\cdot$ Junxing Chen
}

Published online: 16 July 2014

(C) Springer-Verlag Berlin Heidelberg 2014

Erratum to: World J Urol

DOI 10.1007/s00345-014-1342-1

The authors' affiliation was incorrect in the original publication. It did not include the vital Grant information as well.

The correct affiliation details and the funding information are mentioned here.

Acknowledgments The project was supported by Guangdong Natural Science Foundation (S2012040011125, S2012010010964).

The online version of the original article can be found under doi:10.1007/s00345-014-1342-1.

X. Chen $\cdot$ J. Liao $\cdot$ L. Chen $\cdot$ S. Qiu $\cdot$ C. Mo $\cdot$ X. Mao

Y. Yang · S. Zhou $\cdot$ J. Chen $(\varangle)$

Department of Urology, The First Affiliated Hospital,

Sun Yat-Sen University, Guangzhou, China

e-mail: junxingchen@hotmail.com 